TAMKANG JOURNAL OF MATHEMATICS

Volume 33, Number 1, Spring 2002

\title{
MULTIVALUED IMPULSIVE NEUTRAL FUNCTIONAL DIFFERENTIAL INCLUSIONS IN BANACH SPACES
}

\author{
M. BENCHOHRA, J. HENDERSON AND S. K. NTOUYAS
}

\begin{abstract}
In paper the existence of solutions for first and second order impulsive neutral functional differential inclusions in Banach spaces is investigated. The results are obtained by using a fixed point theorem for condensing multivalued maps due to Martelli and the semigroup theory.
\end{abstract}

\section{Introduction}

The necessity of study of impulsive functional differential equations is caused by the fact that they are an adequate mathematical apparatus for simulation of processes and phenomena subject to short-time perturbations during their evolution. The perturbations are performed discretely and their duration is negligible in comparison with the total duration of the processes and phenomena. That is why they are considered to take place "momentarily" in the form of impulses. The theory of impulsive differential equations has been developing considerably, see the monographs of Bainov and Simeonov [1], Lakshmikantham et al [12], and Samoilenko and Perestyuk [16] where numerous properties of their solutions are studied, and a detailed bibliography is given.

Section 3 is concerned with the existence of solutions for the first order initial value problem for neutral functional differential inclusions with impulsive effects as

$$
\begin{gathered}
\frac{d}{d t}\left[y(t)-g\left(t, y_{t}\right)\right] \in F\left(t, y_{t}\right), \quad t \in J=[0, T], t \neq t_{k}, \quad k=1, \ldots, m, \\
\left.\Delta y\right|_{t=t_{k}}=I_{k}\left(y\left(t_{k}^{-}\right)\right), \quad k=1, \ldots, m, \\
y(t)=\phi(t), \quad t \in[-r, 0]
\end{gathered}
$$

where $F: J \times C([-r, 0], E) \longrightarrow 2^{E}$ is a bounded, closed and convex valued multivalued map, $g: J \times C([-r, 0], E) \longrightarrow E$ is a given function, $\phi \in C([-r, 0], E),(0<r<\infty), 0=$ $t_{0}<t_{1}<\ldots<t_{m}<t_{m+1}=T, I_{k} \in C(E, E)(k=1,2, \ldots, m)$, are bounded functions, $\left.\Delta y\right|_{t=t_{k}}=y\left(t_{k}^{+}\right)-y\left(t_{k}^{-}\right), y\left(t_{k}^{-}\right)$and $y\left(t_{k}^{+}\right)$represent the left and right limits of $y(t)$ at $t=t_{k}$, respectively and $E$ a real Banach space with norm $|\cdot|$.

Received March 22, 2001.

2000 Mathematics Subject Classification. 34A37, 34A60, 34G20, 34K25.

Key words and phrases. Impulsive neutral functional differential inclusions, fixed point, Banach space. 
For any continuous function $y$ defined on $[-r, T]-\left\{t_{1}, \ldots, t_{m}\right\}$ and any $t \in J$, we denote by $y_{t}$ the element of $C([-r, 0], E)$ defined by $y_{t}(\theta)=y(t+\theta), \quad \theta \in[-r, 0]$. Here $y_{t}(\cdot)$ represents the history of the state from time $t-r$, up to the present time $t$.

In Section 4 we study second order impulsive neutral functional differential inclusions of the form

$$
\begin{gathered}
\frac{d}{d t}\left[y^{\prime}(t)-g\left(t, y_{t}\right)\right] \in F\left(t, y_{t}\right), \quad t \in J=[0, T], t \neq t_{k}, \quad k=1, \ldots, m, \\
\left.\Delta y\right|_{t=t_{k}}=I_{k}\left(y\left(t_{k}^{-}\right)\right), \quad k=1, \ldots, m, \\
\left.\Delta y^{\prime}\right|_{t=t_{k}}=\bar{I}_{k}\left(y\left(t_{k}^{-}\right)\right), \quad k=1, \ldots, m, \\
y(t)=\phi(t), \quad t \in[-r, 0], y^{\prime}(0)=\eta
\end{gathered}
$$

where $f, g, I_{k}, \bar{I}_{k}$ and $\phi$ are as in problem (1.1)-(1.3), $\bar{I}_{k} \in C(E, E)$ and $\eta \in E$.

Recently an extension to functional differential inclusions with impulsive effects has been done by Benchohra et al [3], [4] with the aid of the nonlinear alternative for multivalued maps and a fixed point theorem for condensing multivalued maps due to Martelli. Other results on functional differential equations without impulsive effect can be found in the monograph of Erbe et al [7], Hale and Verduyn Lunel [9], Henderson [10], and the survey paper of Ntouyas [15].

This paper will be divided into four sections. In Section 2 we will recall briefly some basic definitions and preliminary facts which will be used throughout Sections 3 and 4 . In sections 3 and 4 we shall establish existence theorems for (1.1)-(1.3) and (1.4)-(1.7). Our approach is based on a fixed point theorem for condensing multivalued maps due to Martelli [14]. The results of the present paper extend and generalize some results in the literature on neutral functional differential inclusions in the absence of the impulsive effect. They are also an extension to the multivalued case of similar problems considered by the authors [5], in the single valued case, where the Schaefer's fixed point theorem and the semigroup theory are used.

\section{Preliminaries}

In this section, we introduce notations, definitions, and preliminary facts which are used throughout this paper.

$C([-r, 0], E)$ is the Banach space of all continuous functions from $[-r, 0]$ into $E$ with the norm

$$
\|\phi\|=\sup \{|\phi(\theta)|:-r \leq \theta \leq 0\} .
$$

By $C(J, E)$ we denote the Banach space of all continuous functions from $J$ into $E$ with the norm

$$
\|y\|_{J}:=\sup \{|y(t)|: t \in J\} .
$$

A measurable function $y: J \longrightarrow E$ is Bochner integrable if and only if $|y|$ is Lebesgue integrable. (For properties of the Bochner integral, see for instance, Yosida [17]). 
$L^{1}(J, E)$ denotes the Banach space of functions $y: J \longrightarrow E$ which are Bochner integrable normed by

$$
\|y\|_{L^{1}}=\int_{0}^{T}|y(t)| d t \quad \text { for all } \quad y \in L^{1}(J, E) .
$$

Let $(X,|\cdot|)$ be a Banach space. A multivalued map $G: X \rightarrow 2^{X}$ has convex (closed) values if $G(x)$ is convex (closed) for all $x \in X$. $G$ is bounded on bounded sets if $G(B)$ is bounded in $X$ for each bounded set $B$ of $X$ (i.e. $\sup _{x \in B}\{\sup \{|y|: y \in G(x)\}\}<\infty$ ).

$G$ is called upper semicontinuous (u.s.c.) on $X$ if for each $x_{0} \in X$ the set $G\left(x_{0}\right)$ is a nonempty, closed subset of $X$, and if for each open set $N$ of $X$ containing $G\left(x_{0}\right)$, there exists an open neighbourhood $M$ of $x_{0}$ such that $G(M) \subseteq N$. $G$ is said to be completely continuous if $G(B)$ is relatively compact for every bounded subset $B \subseteq X$.

If the multivalued $G$ is completely continuous with nonempty compact values, then $G$ is u.s.c. if and only if $G$ has a closed graph (i.e. $x_{n} \longrightarrow x_{*}, y_{n} \longrightarrow y_{*}, y_{n} \in G\left(x_{n}\right)$ imply $\left.y_{*} \in G\left(x_{*}\right)\right)$. $G$ has a fixed point if there is $x \in X$ such that $x \in G(x)$.

In the following $C C(E)$ denotes the set of all nonempty compact, convex subsets of $E$. A multivalued map $G: J \rightarrow C C(X)$ is said to be measurable if for each $x \in E$ the function $Y: J \longrightarrow \mathbb{R}$ defined by $Y(t)=d(x, G(t))=\inf \{|x-z|: z \in G(t)\}$ is measurable.

An upper semi-continuous multivalued map $G: X \longrightarrow 2^{X}$ is said to be condensing [2] if for any subset $B \subseteq X$ with $\alpha(B) \neq 0$, we have $\alpha(G(B))<\alpha(B)$, where $\alpha$ denotes the Kuratowski measure of noncompacteness [2].

We remark that a completely continuous multivalued map is the easiest example of a condensing map. For more details on multivalued maps see the books of Deimling [6], Gorniewicz [8] and Hu and Papageorgiou[11].

Definition 2.1. A multivalued map $F: J \times C([-r, 0], E) \rightarrow 2^{E}$ is said to be $L^{1}$-Carathéodory if

(i) $t \longmapsto F(t, u)$ is measurable for each $u \in C([-r, 0], E)$;

(ii) $u \longmapsto F(t, u)$ is upper semicontinuous for almost all $t \in J$;

(iii) For each $q>0$, there exists $l_{q} \in L^{1}\left(J, \mathbb{R}_{+}\right)$such that

$$
\begin{aligned}
& \|F(t, u)\|=\sup \{|v|: v \in F(t, u)\} \leq l_{q}(t) \\
& \quad \text { for all }\|u\| \leq q \text { and for almost all } t \in J .
\end{aligned}
$$

In order to define the solution of $(1,1)-(1.3)$ we shall consider the following spaces

$$
\begin{aligned}
\Omega= & \left\{y:[-r, T] \longrightarrow E: y_{k} \in C\left(J_{k}, E\right), k=0, \ldots, m\right. \text { and there exist } \\
& \left.y\left(t_{k}^{-}\right) \text {and } y\left(t_{k}^{+}\right), k=1, \ldots, m \text { with } y\left(t_{k}^{-}\right)=y\left(t_{k}\right), y(t)=\phi(t), \forall t \in[-r, 0]\right\}
\end{aligned}
$$


which is a Banach space with the norm

$$
\|y\|_{\Omega}=\max \left\{\left\|y_{k}\right\|_{J_{k}}, k=0, \ldots, m\right\}
$$

where $y_{k}$ is the restriction of $y$ to $J_{k}=\left[t_{k}, t_{k+1}\right], k=0, \ldots, m$.

We shall also consider the set

$$
\begin{aligned}
\Omega^{1}= & \left\{y:[-r, T] \longrightarrow E: y_{k} \in W^{1,1}\left(J_{k}, E\right), k=0, \ldots, m\right. \text { and there exist } \\
& \left.y\left(t_{k}^{-}\right) \text {and } y\left(t_{k}^{+}\right), k=1, \ldots, m \text { with } y\left(t_{k}^{-}\right)=y\left(t_{k}\right), y(t)=\phi(t), \forall t \in[-r, 0]\right\} .
\end{aligned}
$$

The set $\Omega^{1}$ is a Banach space with the norm

$$
\|y\|_{\Omega^{1}}=\max \left\{\left\|y_{k}\right\|_{W^{1,1}\left(J_{k}, E\right)}, k=0, \ldots, m\right\} .
$$

So let us start by defining what we mean by a solution of problem (1.1)-(1.3).

Definition 2.2. A function $y \in \Omega \cap \Omega^{1}$ is said to be a solution of (1.1)-(1.3) if $y(t)-g\left(t, y_{t}\right)$ is absolutely continuous on $J-\left\{t_{1}, \ldots, t_{m}\right\}$ and (1.1) to (1.3) are satisfied.

Our main result are based on the following:

Lemma 2.3. [13]. Let $I$ be a compact real interval and $X$ be a Banach space. Let $F$ be a multivalued map satisfying the Carathéodory conditions with the set of $L^{1}$-selections $S_{F}$ nonempty, and let $\Gamma$ be a linear continuous mapping from $L^{1}(I, X)$ to $C(I, X)$. Then the operator

$$
\Gamma \circ S_{F}: C(I, X) \longrightarrow C C(C(I, X)), y \longmapsto\left(\Gamma \circ S_{F}\right)(y):=\Gamma\left(S_{F, y}\right)
$$

is a closed graph operator in $C(I, X) \times C(I, X)$.

Lemma 2.4. [14] Let $N: X \longrightarrow C C(X)$ be a condensing map. If the set

$$
\mathcal{M}:=\{y \in X: \lambda y \in N(y) \text { for some } \lambda>1\}
$$

is bounded, then $N$ has a fixed point.

Let $v \in L^{1}(J, E)$ and consider the IVP (2.1), (1.2), (1.3)

$$
\frac{d}{d t}\left[y(t)-g\left(t, y_{t}\right)\right]=v(t), \quad t \in J, t \neq t_{k}, \quad k=1, \ldots, m .
$$

We need the following auxiliary result. Its proof is very simple, so we omit it.

Lemma 2.5. $y \in \Omega^{1}$ is a solution of (2.1), (1.2), (1.3) if and only if $y \in \Omega$ is a solution of the impulsive integral equation

$$
y(t)=\phi(0)-g(0, \phi(0))+g\left(t, y_{t}\right)+\int_{0}^{t} v(s) d s+\sum_{0<t_{k}<t} I_{k}\left(y\left(t_{k}^{-}\right)\right) \text {for } t \in J .
$$




\section{First Order Impulsive NFDIs}

We are now in a position to state and prove our existence result for the problem (1.1)-(1.3). For the study of this problem we first list the following hypotheses:

(H1) $F: J \times C([-r, 0], E) \longrightarrow B C C(E)$ is an $L^{1}$-Carathéodory map;

(H2) There exist constants $0 \leq c_{1}<1$ and $c_{2} \geq 0$ such that

$$
|g(t, u)| \leq c_{1}\|u\|+c_{2}, \quad t \in J, u \in C([-r, 0], E)
$$

(H3) There exist constants $d_{k}$ such that $\left|I_{k}(y)\right| \leq d_{k}, k=1, \ldots, m$ for each $y \in E$;

(H4) $\|F(t, u)\|=\sup \{|v|: v \in F(t, u)\} \leq p(t) \psi(\|u\|)$ for almost all $t \in J$ and all $u \in C([-r, 0], E)$, where $p \in L^{1}\left(J, \mathbb{R}_{+}\right)$and $\psi: \mathbb{R}_{+} \longrightarrow(0, \infty)$ is continuous and increasing with

$$
\frac{1}{1-c_{1}} \int_{0}^{T} p(s) d s<\int_{c}^{\infty} \frac{d \tau}{\psi(\tau)}
$$

where $c=\frac{1}{1-c_{1}}\left\{\left(1+c_{1}\right)\|\phi\|+2 c_{2}+\sum_{k=1}^{m} d_{k}\right\}$;

(H5) The function $g$ is completely continuous and for any bounded set $A \subseteq \Omega$ the set $\left\{t \rightarrow g\left(t, y_{t}\right): y \in A\right\}$ is equicontinuous in $\Omega$;

(H6) For each bounded $B \subseteq \Omega$ and $t \in J$ the set

$$
\left\{\phi(0)+\int_{0}^{t} v(s) d s+\sum_{0<t_{k}<t} I_{k}\left(y\left(t_{k}\right)\right): v \in S_{F, B}\right\}
$$

is relatively compact in $E$, where $S_{F, B}=\cup\left\{S_{F, y}: y \in B\right\}$.

Remark 3.1. (i) If $\operatorname{dim} E<\infty$, then for each $u \in C([-r, 0], E)$ the set $S_{F, u}$ is nonempty (see Lasota and Opial [13]).

(ii) If $\operatorname{dim} E=\infty$ and $u \in C([-r, 0], E)$ the set $S_{F, u}$ is nonempty if and only if the function $Y: J \longrightarrow \mathbb{R}$ defined by

$$
Y(t):=\inf \{|v|: v \in F(t, u)\}
$$

belongs to $L^{1}(J, \mathbb{R})$ (see Hu and Papageorgiou [11]).

Theorem 3.2. Assume that hypotheses (H1)-(H6) hold. Then the IVP (1.1)-(1.3) has at least one solution on $[-r, T]$. 
Proof. Transform the problem into a fixed point problem. Consider the operator, $N: \Omega \longrightarrow 2^{\Omega}$ defined by:

$$
N(y)=\left\{h \in \Omega: h(t)\left\{\begin{array}{ll}
\phi(t), & t \in[-r, 0] \\
\phi(0)-g(0, \phi(0))+g\left(t, y_{t}\right) & \\
+\int_{0}^{t} v(s) d s+\sum_{0<t_{k}<t} I_{k}\left(y\left(t_{k}\right)\right), t \in J,
\end{array}\right\}\right.
$$

where $v \in S_{F, y}$.

Remark 3.3. Clearly from Lemma (2.5) the fixed points of $N$ are solutions to (1.1)-(1.3).

We shall show that $N$ satisfies the assumptions of Lemma 2.4. Using (H5) it suffices to show that the operator $N_{1}: \Omega \longrightarrow 2^{\Omega}$ defined by:

$$
N_{1}(y)=\left\{h \in \Omega: h(t)=\left\{\begin{array}{ll}
\phi(t), & t \in[-r, 0] \\
\phi(0)+\int_{0}^{t} v(s) d s+\sum_{0<t_{k}<t} I_{k}\left(y\left(t_{k}\right)\right), t \in J,
\end{array}\right\}\right.
$$

where $v \in S_{F, y}$, is u.s.c. condensing with bounded, closed and convex values. The proof will be given in several steps.

Step 1. $N_{1}(y)$ is convex for each $y \in \Omega$.

This is obvious since $S_{F, y}$ is convex (because $F$ has convex values).

Step 2. $N_{1}$ maps bounded sets into relatively compact sets in $\Omega$.

This is a consequence of the $L^{1}$-Carathéodory character of $F$. As a consequence of Steps 1 and 2 and (H4) together with the Arzela-Ascoli theorem we can conclude that $N: \Omega \longrightarrow 2^{\Omega}$ is completely continuous multivalued and therefore, a condensing map.

Step 4. $N$ has a closed graph.

Let $y_{n} \longrightarrow y_{*}, h_{n} \in N\left(y_{n}\right)$, and $h_{n} \longrightarrow h_{*}$. We shall prove that $h_{*} \in N\left(y_{*}\right)$. $h_{n} \in N\left(y_{n}\right)$ means that there exists $v_{n} \in S_{F, y_{n}}$ such that for each $t \in J$

$$
h_{n}(t)=\phi(0)-g(0, \phi(0))+g\left(t, y_{n t}\right)+\int_{0}^{t} v_{n}(s) d s+\sum_{0<t_{k}<t} I_{k}\left(y_{n}\left(t_{k}\right)\right) .
$$

We must prove that there exists $v_{*} \in S_{F, y_{*}}$ such that for each $t \in J$

$$
h_{*}(t)=\phi(0)-g(0, \phi(0))+g\left(t, y_{* t}\right)+\int_{0}^{t} v_{*}(s) d s+\sum_{0<t_{k}<t} I_{k}\left(y_{*}\left(t_{k}\right)\right) .
$$

Since the functions $g(t,),. t \in J, I_{k}, k=1, \ldots, m$ are continuous we have that

$$
\begin{aligned}
& \|\left(h_{n}-\phi(0)+g(0, \phi(0))-g\left(t, y_{n t}\right)-\sum_{0<t_{k}<t} I_{k}\left(y_{n}\left(t_{k}\right)\right)\right) \\
& \quad-\left(h_{*}-\phi(0)+g(0, \phi(0))-g\left(t, y_{* t}\right)-\sum_{0<t_{k}<t} I_{k}\left(y_{*}\left(t_{k}\right)\right)\right) \|_{\Omega} \longrightarrow 0, \text { as } n \rightarrow \infty .
\end{aligned}
$$


Consider the linear continuous operator

$$
\begin{gathered}
\Gamma: L^{1}(J, E) \longrightarrow C(J, E) \\
v \longmapsto \Gamma(v)(t)=\int_{0}^{t} v(s) d s .
\end{gathered}
$$

From Lemma 2.3, it follows that $\Gamma \circ S_{F}$ is a closed graph operator.

Moreover, we have that

$$
\left(h_{n}(t)-\phi(0)+g(0, \phi(0))-g\left(t, y_{n t}\right)-\sum_{0<t_{k}<t} I_{k}\left(y_{n}\left(t_{k}\right)\right)\right) \in \Gamma\left(S_{F, y_{n}}\right) .
$$

Since $y_{n} \longrightarrow y_{*}$, it follows from Lemma 2.3 that

$$
\left(h_{*}(t)-\phi(0)+g(0, \phi(0))-g\left(t, y_{* t}\right)-\sum_{0<t_{k}<t} I_{k}\left(y_{*}\left(t_{k}\right)\right)\right)=\int_{0}^{t} v_{*}(s) d s
$$

for some $g_{*} \in S_{F, y_{*}}$.

Step 5. Now it remains to show that the set

$$
\mathcal{M}:=\{y \in \Omega: \lambda y \in N(y), \text { for some } \lambda>1\}
$$

is bounded.

Let $y \in \mathcal{M}$. Then $y \in \lambda N(y)$ for some $0<\lambda<1$. Thus for each $t \in J$

$$
y(t)=\lambda^{-1} \phi(0)-\lambda^{-1} g(0, \phi(0))+\lambda^{-1} g\left(t, y_{t}\right)+\lambda^{-1} \int_{0}^{t} v(s) d s+\lambda^{-1} \sum_{0<t_{k}<t} I_{k}\left(y\left(t_{k}\right)\right) .
$$

This implies by (H2)-(H4) that for each $t \in J$ we have

$$
|y(t)| \leq\|\phi\|+c_{1}\|\phi\|+2 c_{2}+c_{1}\left\|y_{t}\right\|+\int_{0}^{t} p(s) \psi\left(\left\|y_{s}\right\|\right) d s+\sum_{k=1}^{m} d_{k} .
$$

We consider the function $\mu$ defined by

$$
\mu(t)=\sup \{|y(s)|:-r \leq s \leq t\}, \quad 0 \leq t \leq T .
$$

Let $t^{*} \in[-r, t]$ be such that $\mu(t)=\left|y\left(t^{*}\right)\right|$. If $t^{*} \in[0, T]$, by the previous inequality we have for $t \in[0, T]$

$$
\begin{aligned}
\mu(t) & \leq\|\phi\|+c_{1}\|\phi\|+2 c_{2}+c_{1}\left\|y_{t}\right\|+\int_{0}^{t} p(s) \psi\left(\left\|y_{s}\right\|\right) d s+\sum_{0<t_{k}<t}\left|I_{k}\left(y\left(t_{k}\right)\right)\right| \\
& \leq\|\phi\|+c_{1}\|\phi\|+2 c_{2}+c_{1} \mu(t)+\int_{0}^{t} p(s) \psi(\mu(s)) d s+\sum_{k=1}^{m} d_{k} .
\end{aligned}
$$


Thus

$$
\mu(t) \leq \frac{1}{1-c_{1}}\left\{\left(1+c_{1}\right)\|\phi\|+2 c_{2}+\int_{0}^{t} p(s) \psi(\mu(s)) d s+\sum_{k=1}^{m} d_{k}\right\} .
$$

If $t^{*} \in J_{0}$ then $\mu(t)=\|\phi\|$ and the previous inequality holds.

Let us take the right-hand side of the above inequality as $v(t)$, then we have

$$
c=v(0)=\frac{1}{1-c_{1}}\left\{\left(1+c_{1}\right)\|\phi\|+2 c_{2}+\sum_{k=1}^{m} d_{k}\right\}, \quad \mu(t) \leq v(t), \quad t \in J
$$

and

$$
v^{\prime}(t)=\frac{1}{1-c_{1}} p(t) \psi(\mu(t)), \quad t \in J
$$

Using the nondecreasing character of $\psi$ we get

$$
v^{\prime}(t) \leq \frac{1}{1-c_{1}} p(t) \psi(v(t)), \quad t \in J .
$$

This implies for each $t \in J$ that

$$
\int_{v(0)}^{v(t)} \frac{d u}{\psi(u)} \leq \frac{1}{1-c_{1}} \int_{0}^{T} p(s) d s<\int_{v(0)}^{\infty} \frac{d u}{\psi(u)} .
$$

This inequality implies that there exists a constant $b$ such that $v(t) \leq b, t \in J$, and hence $\mu(t) \leq b, t \in J$. Since for every $t \in[0, T],\left\|y_{t}\right\| \leq \mu(t)$, we have

$$
\|y\|_{\Omega} \leq b^{\prime}=\operatorname{Max}\{\|\phi\|, b\},
$$

where $b^{\prime}$ depends only $T$ and on the functions $p$ and $\psi$. This shows that $\mathcal{M}$ is bounded.

Set $X:=\Omega$. As a consequence of Lemma 2.4 we deduce that $N$ has a fixed point which is a solution of (1.1)-(1.3).

\section{Second Order Impulsive NFDIs}

In this section we study the initial value problem (1.4)-(1.7).

Definition 4.1. A function $y \in \Omega \cap \bar{\Omega}^{1}$ is said to be a solution of (1.4)-(1.7) if $y$ and $y^{\prime}(t)-g\left(t, y_{t}\right)$ are absolutely continuous on $J-\left\{t_{1}, \ldots, t_{m}\right\}$ and (1.4) to (1.7) are satisfied.

Here

$$
\begin{aligned}
\bar{\Omega}^{1}= & \left\{y:[-r, T] \longrightarrow E: y_{k} \in W^{2,1}\left(J_{k}, E\right), k=0, \ldots, m\right. \text { and there exist } \\
& \left.y\left(t_{k}^{-}\right) \text {and } y\left(t_{k}^{+}\right), k=1, \ldots, m \text { with } y\left(t_{k}^{-}\right)=y\left(t_{k}\right), y(t)=\phi(t), \forall t \in[-r, 0]\right\}
\end{aligned}
$$

which is a Banach space with the norm

$$
\|y\|_{\bar{\Omega}^{1}}=\operatorname{Max}\left\{\left\|y_{k}\right\|_{W^{2,1}\left(J_{k}, E\right)}, k=0, \ldots, m\right\} .
$$

Theorem 4.2. Assume that hypotheses (H1), (H2) (with $\left.c_{1} \geq 0\right),(H 5)$ and 
(H3)' There exist constants $d_{k}, \bar{d}_{k}$ such that $\left|I_{k}(y)\right| \leq d_{k},\left|\bar{I}_{k}(y)\right| \leq \bar{d}_{k} k=1, \ldots, m$ for each $y \in E$;

(H4)' $\|F(t, u)\| \leq p(t) \psi(\|u\|)$ for almost all $t \in J$ and all $u \in C([-r, 0], E)$, where $p \in$ $L^{1}\left(J, \mathbb{R}_{+}\right)$and $\psi: \mathbb{R}_{+} \longrightarrow(0, \infty)$ is continuous and increasing with

$$
\int_{0}^{T} M(s) d s<\int_{\bar{c}}^{\infty} \frac{d s}{s+\psi(s)}
$$

where $\bar{c}=\|\phi\|+\left[|\eta|+c_{1}\|\phi\|+2 c_{2}\right] T+\sum_{k=1}^{m}\left[d_{k}+\left(T-t_{k}\right) \bar{d}_{k}\right]$, and $M(t)=\max \left\{1, c_{1}, p(t)\right\}$.

(H6)' For each bounded $B \subseteq \Omega$ and $t \in J$ the set

$$
\left\{\phi(0)+t \eta+\int_{0}^{t} \int_{0}^{s} v(u) d u d s+\sum_{0<t_{k}<t}\left[I_{k}\left(y\left(t_{k}\right)\right)+\left(t-t_{k}\right) \bar{I}_{k}\left(y\left(t_{k}\right)\right)\right]: v \in S_{F, B}\right\}
$$

is relatively compact in $E$, where $S_{F, B}=\cup\left\{S_{F, y}: y \in B\right\}$

hold. Then the IVP (1.4)-(1.7) has at least one solution on $[-r, T]$.

Proof. Transform the problem into a fixed point problem. Consider the operator, $N^{\prime}: \Omega \longrightarrow \Omega$ defined by:

$$
N^{\prime}(y)=\left\{h \in \Omega: h(t)\left\{\begin{array}{ll}
\phi(t), & t \in[-r, 0] \\
\phi(0)+[\eta-g(0, \phi(0))] t & t \\
+\int_{0}^{t} g\left(s, y_{s}\right) d s+\int_{0}^{t} \int_{0}^{u} v(u) d u d s \\
+\sum_{0<t_{k}<t}\left[I_{k}\left(y\left(t_{k}\right)\right)+\left(t-t_{k}\right) \bar{I}_{k}\left(y\left(t_{k}\right)\right)\right], t \in J,
\end{array}\right\}\right.
$$

where $v \in S_{F, y}$. As in Theorem 3.2, we can prove that $N^{\prime}$ is a bounded, closed and convex valued multivalued map and is u.s.c. We omit the details. Here we repeat only the proof that the set

$$
\Phi\left(N^{\prime}\right):=\left\{y \in \Omega: y=\lambda N^{\prime}(y), \text { for some } 0<\lambda<1\right\}
$$

is bounded.

Let $y \in \Phi\left(N^{\prime}\right)$. Then $y=\lambda N^{\prime}(y)$ for some $0<\lambda<1$. Thus for each $t \in J$

$$
\begin{aligned}
y(t)= & \lambda^{-1} \phi(0)+\lambda^{-1}[\eta-g(0, \phi(0))] t+\lambda^{-1} \int_{0}^{t} g\left(s, y_{s}\right) d s+\lambda^{-1} \int_{0}^{t} \int_{0}^{u} v(u) d u d s \\
& +\lambda^{-1} \sum_{0<t_{k}<t}\left[I_{k}\left(y\left(t_{k}\right)\right)+\left(t-t_{k}\right) \bar{I}_{k}\left(y\left(t_{k}\right)\right)\right] .
\end{aligned}
$$


This implies by (H2), (H3)' and (H4)' that for each $t \in J$ we have

$$
\begin{aligned}
|y(t)| \leq & \|\phi\|+\left[|\eta|+c_{1}\|\phi\|+2 c_{2}\right] T+c_{1} \int_{0}^{t}\left\|y_{s}\right\| d s \\
& +\int_{0}^{t} \int_{0}^{s} p(u) \psi\left(\left\|y_{u}\right\|\right) d u d s+\sum_{k=1}^{m}\left[d_{k}+\left(T-t_{k}\right) \bar{d}_{k}\right] \\
\leq & \|\phi\|+\left[|\eta|+c_{1}\|\phi\|+2 c_{2}\right] T+c_{1} \int_{0}^{t} M(s)\left\|y_{s}\right\| d s \\
& +\int_{0}^{t} M(s) \int_{0}^{s} p(u) \psi\left(\left\|y_{u}\right\|\right) d u d s+\sum_{k=1}^{m}\left[d_{k}+\left(T-t_{k}\right) \bar{d}_{k}\right],
\end{aligned}
$$

where $M(t)=\max \left\{1, c_{1}, p(t)\right\}$. We consider the function $\mu$ defined by

$$
\mu(t)=\sup \{|y(s)|:-r \leq s \leq t\}, \quad 0 \leq t \leq T .
$$

Let $t^{*} \in[-r, t]$ be such that $\mu(t)=\left|y\left(t^{*}\right)\right|$. If $t^{*} \in[0, T]$, by the previous inequality we have for $t \in[0, T]$

$$
\begin{aligned}
\mu(t) \leq & \|\phi\|+\left[|\eta|+c_{1}\|\phi\|+2 c_{2}\right] T+\int_{0}^{t} M(s) \mu(s) d s \\
& +\int_{0}^{t} M(s) \int_{0}^{s} \psi(\mu(u)) d u d s+\sum_{k=1}^{m}\left[d_{k}+\left(T-t_{k}\right) \bar{d}_{k}\right] .
\end{aligned}
$$

If $t^{*} \in J_{0}$ then $\mu(t)=\|\phi\|$ and the previous inequality holds.

Let us take the right-hand side of the above inequality as $v(t)$, then we have

$$
c=v(0)=\|\phi\|+\left[|\eta|+c_{1}\|\phi\|+2 c_{2}\right] T+\sum_{k=1}^{m}\left[d_{k}+\left(T-t_{k}\right) \bar{d}_{k}\right], \quad \mu(t) \leq v(t), \quad t \in J
$$

and

$$
\begin{aligned}
v^{\prime}(t) & =M(t) \mu(t)+M(t) \int_{0}^{t} \psi(\mu(t)) \\
& \leq M(t)\left[v(t)+\int_{0}^{t} \psi(v(t))\right], \quad t \in J
\end{aligned}
$$

Put

$$
u(t)=v(t)+\int_{0}^{t} \psi(v(s)) d s, \quad t \in J .
$$

Then

$$
u(0)=v(0)=c, \quad v(t) \leq u(t), \quad t \in J
$$


and

$$
u^{\prime}(t)=v^{\prime}(t)+\psi(v(t)) \leq M(t)[u(t)+\psi(u(t))], \quad t \in J .
$$

This implies for each $t \in J$ that

$$
\int_{u(0)}^{u(t)} \frac{d u}{u+\psi(u)} \leq \int_{0}^{T} M(s) d s<\int_{u(0)}^{\infty} \frac{d u}{u+\psi(u)} .
$$

This inequality implies that there exists a constant $b_{1}$ such that $u(t) \leq b_{1}, t \in J$, and hence $\mu(t) \leq b_{1}, t \in J$. Since for every $t \in[0, T],\left\|y_{t}\right\| \leq \mu(t)$, we have

$$
\|y\|_{\Omega} \leq b^{\prime \prime}=\operatorname{Max}\left\{\|\phi\|, b_{1}\right\},
$$

where $b^{\prime \prime}$ depends only $T$ and on the functions $p$ and $\psi$. This shows that $\Phi\left(N^{\prime}\right)$ is bounded.

\section{References}

[1] D. D. Bainov and P. S. Simeonov, Systems with Impulse Effect, Ellis Horwood Ltd., Chichister, 1989.

[2] J. Banas and K. Goebel, Measures of Noncompactness in Banach Spaces, Marcel-Dekker, New York, 1980.

[3] M. Benchohra, J. Henderson and S. K. Ntouyas, Impulsive functional differential inclusions in Banach spaces, Commu. Appl. Anal. (to appear).

[4] M. Benchohra, J. Henderson and S. K. Ntouyas, On a boundary value problem for second order impulsive functional differential inclusion in Banach spaces, Intern. J. Nonlinear Differ. Equations (to appear).

[5] M. Benchohra, J. Henderson and S. K. Ntouyas, Impulsive neutral functional differential equations in Banach spaces. (submitted).

[6] K. Deimling, Multivalued Differential Equations, Walter De Gruyter, Berlin-New York, 1992.

[7] L. H. Erbe, Q. Kong and B. G. Zhang, Oscillation Theory for Functional Differential Equations, Pure and Applied Mathematics, Marcel Dekker, 1994.

[8] L. Gorniewicz, Topological Fixed Point Theory of Multivalued Mappings, Mathematics and its Applications, 495, Kluwer Academic Publishers, Dordrecht, 1999.

[9] J. K. Hale and S. M. Verduyn Lunel, Introduction to Functional Differential Equations, Springer Verlag, New York, Berlin, 1993.

[10] J. Henderson, Boundary Value Problems for Functional Differenial Equations, World Scientific, Singapore, 1995.

[11] Sh. Hu and N. S. Papageorgiou, Handbook of Multivalued Analysis, Volume I: Theory, Kluwer, Dordrecht, Boston, London, 1997.

[12] V. Lakshmikantham, D. D. Bainov and P. S. Simeonov, Theory of Impulsive Differential Equations, World Scientific, Singapore, 1989.

[13] A. Lasota and Z. Opial, An application of the Kakutani-Ky Fan theorem in the theory of ordinary differential equations, Bull. Acad. Pol. Sci. Ser. Sci. Math. Astronom. Phys., 13 (1965), 781-786. 
[14] M. Martelli, A Rothe's type theorem for non-compact acyclic-valued map, Boll. Un. Mat. Ital., 4 (1975), 70-76.

[15] S. K. Ntouyas, Initial and boundary value problems for functional differential equations via the topological transversality method: A survey, Bull. Greek Math. Soc., 40 (1998), 3-41.

[16] A. M. Samoilenko and N. A. Perestyuk, Impulsive Differential Equations, World Scientific, Singapore, 1995.

[17] K. Yosida, Functional Analysis, $6^{\text {th }}$ edn. Springer-Verlag, Berlin, 1980.

[18] D. Yujun, Periodic boundary value problems for functional differential equations with impulses, J. Math. Anal. Appl., 210 (1997), 170-181.

Department of Mathematics, University of Sidi Bel Abbes, BP 89, 22000 Sidi Bel Abbes, Algeria.

E-mail address: benchohra@yahoo.com

Department of Mathematics, Auburn University, Auburn, Alabama 36849-5310 USA.

E-mail address: hendej2@mail.auburn.edu

Department of Mathematics, University of Ioannina, 45110 Ioannina, Greece.

E-mail address: sntouyas@cc.uoi.gr 